\title{
Uniqueness in Discrete Tomography of Delone Sets with Long-Range Order
}

\author{
Christian Huck
}

Received: 27 November 2007 / Revised: 31 October 2008 / Accepted: 3 February 2009 /

Published online: 17 July 2009

(C) Springer Science+Business Media, LLC 2009

\begin{abstract}
We address the problem of determining finite subsets of Delone sets $\Lambda \subset \mathbb{R}^{d}$ with long-range order by $X$-rays in prescribed $\Lambda$-directions, i.e., directions parallel to nonzero interpoint vectors of $\Lambda$. Here, an $X$-ray in direction $u$ of a finite set gives the number of points in the set on each line parallel to $u$. For our main result, we introduce the notion of algebraic Delone sets $\Lambda \subset \mathbb{R}^{2}$ and derive a sufficient condition for the determination of the convex subsets of these sets by $X$-rays in four prescribed $\Lambda$-directions.
\end{abstract}

Keywords Discrete tomography $\cdot$ Discrete parallel $X$-ray $\cdot U$-polygon - Algebraic Delone set $\cdot p$-adic valuation . Cyclotomic model set

\section{Introduction}

Discrete tomography is concerned with the inverse problem of retrieving information about some finite object from (generally noisy) information about its slices. An important problem is the reconstruction of a finite point set from its line sum functions in a small number of directions. More precisely, a (discrete parallel) $X$-ray of a finite subset of $\mathbb{R}^{d}$ in direction $u$ gives the number of points in the set on each line parallel to $u$. In the traditional setting, motivated by crystals, the positions to be determined

The author was supported by the German Research Council (Deutsche Forschungsgemeinschaft), within the CRC 701, and by EPSRC via Grant EP/D058465/1.

C. Huck $(\bowtie)$

Fakultät für Mathematik, Universität Bielefeld, Postfach 100131, 33501, Bielefeld, Germany

e-mail: huck@math.uni-bielefeld.de

C. Huck

Department of Mathematics and Statistics, The Open University, Walton Hall, Milton Keynes, MK7 6AA, UK

e-mail: c.huck@open.ac.uk 
form a subset of a common translate of a lattice in $\mathbb{R}^{d}$. In fact, many of the problems in discrete tomography have been studied on the square lattice; see [12-14, 17] and [19]. In the longer run, by also having other structures than perfect crystals in mind, one has to take into account wider classes of sets, or at least significant deviations from the lattice structure. As an intermediate step between periodic and random (or amorphous) Delone sets, we consider Delone sets with long-range order, thus including systems of aperiodic order like model sets (also called mathematical quasicrystals) as a special case.

The main motivation for our interest in the discrete tomography of Delone sets $\Lambda$ with long-range order comes from the fact that these sets serve as a rather general model of atomic positions in solid-state materials together with the demand of materials science to reconstruct such three-dimensional structures or planar layers of them from their images under quantitative high-resolution transmission electron microscopy (HRTEM). In fact, in [25] and [34] a technique is described, which, for some crystals, can effectively measure the number of atoms lying on densely occupied lines. Therefore, only $\Lambda$-directions, i.e., directions parallel to nonzero interpoint vectors of $\Lambda$, will be considered. Further, since typical objects may be damaged or even destroyed by the radiation energy after about 3 to 5 images taken by HRTEM, one is restricted to a small number of $X$-rays. It actually is this restriction to few high-density directions that makes the problems of discrete tomography mathematically challenging, even if one assumes the absence of noise.

Since the above reconstruction problem of discrete tomography can possess rather different solutions, one is led to the determination of finite subsets of a fixed Delone set $\Lambda$ by $X$-rays in a small number of suitably prescribed $\Lambda$-directions. More precisely, we say that the elements of a collection $\mathcal{E}$ of finite sets are determined by the $X$-rays in a finite set of directions if different sets $F$ and $F^{\prime}$ in $\mathcal{E}$ cannot have the same $X$-rays in these directions.

After summarizing a few general results on determination, we introduce the class of algebraic Delone sets $\Lambda \subset \mathbb{R}^{2}$ (see Definition 4.1) and study the determination of the convex subsets of these sets by $X$-rays in $\Lambda$-directions. They are finite subsets of $\Lambda$ with the property that their convex hull contains no new points of $\Lambda$. By using $p$-adic valuation methods as introduced in discrete tomography by Gardner and Gritzmann in [12] together with standard facts from the theory of (cyclotomic) fields, we derive a sufficient condition for the determination of the convex subsets of $\Lambda$ by $X$-rays in four $\Lambda$-directions and show that three $\Lambda$-directions never suffice for this purpose; cf. Theorem 4.21. Further, by using standard facts from algebraic number theory and the theory of Pisot-Vijayaraghavan numbers, it is shown that cyclotomic model sets (see Definition 4.22) are examples of algebraic Delone sets; cf. Proposition 4.31. We conclude with a discussion of the result on the determination of convex sets by four $X$-rays for this specific class of objects; cf. Theorem 4.33.

\section{Preliminaries and Notation}

Natural numbers are always assumed to be positive, and we denote by $\mathbb{P}$ the set of rational primes. We denote the norm in Euclidean $d$-space $\mathbb{R}^{d}$ by $\|\cdot\|$. The unit sphere 
in $\mathbb{R}^{d}$ is denoted by $\mathbb{S}^{d-1}$, and its elements are also called directions. For $r>0$ and $x \in \mathbb{R}^{d}, B_{r}(x)$ is the open ball of radius $r$ about $x$. For a subset $S \subset \mathbb{R}^{d}$, a direction $u \in \mathbb{S}^{d-1}$ is called an $S$-direction if it is parallel to a nonzero element of the difference set $S-S$ of $S$. A homothety $h: \mathbb{R}^{d} \rightarrow \mathbb{R}^{d}$ is given by $z \mapsto \lambda z+t$, where $\lambda \in \mathbb{R}$ is positive and $t \in \mathbb{R}^{d}$. A convex polygon is the convex hull of a finite set of points in $\mathbb{R}^{2}$. For a subset $S \subset \mathbb{R}^{2}$, a polygon in $S$ is a convex polygon with all vertices in $S$. Further, a bounded subset $C$ of $S$ is called a convex subset of $S$ if its convex hull contains no new points of $S$, i.e., if $C=\operatorname{conv}(C) \cap S$. Let $U \subset \mathbb{S}^{1}$ be a finite set of directions. A nondegenerate convex polygon $P$ is called a $U$-polygon if it has the property that whenever $v$ is a vertex of $P$ and $u \in U$, the line in the plane in direction $u$ which passes through $v$ also meets another vertex $v^{\prime}$ of $P$. Occasionally, we identify $\mathbb{C}$ with $\mathbb{R}^{2}$, and $\bar{z}$ will always denote the complex conjugate of $z$. Consider a set $\Lambda \subset \mathbb{R}^{d}$, where $d \in \mathbb{N}$. $\Lambda$ is called uniformly discrete if there is a radius $r>0$ such that every ball $B_{r}(x)$ with $x \in \mathbb{R}^{d}$ contains at most one point of $\Lambda$. Note that the bounded subsets of a uniformly discrete set $\Lambda$ are precisely the finite subsets of $\Lambda$. $\Lambda$ is called relatively dense if there is a radius $R>0$ such that every ball $B_{R}(x)$ with $x \in \mathbb{R}^{d}$ contains at least one point of $\Lambda$. $\Lambda$ is called a Delone set if it is both uniformly discrete and relatively dense. $\Lambda$ is said to be of finite local complexity if $\Lambda-\Lambda$ is discrete and closed. Note that $\Lambda$ has finite local complexity if and only if for every $r>0$, there are, up to translation, only finitely many patches of radius $r$, i.e., sets of the form $\Lambda \cap B_{r}(x)$, where $x \in \mathbb{R}^{d}$; cf. [29]. A Delone set $\Lambda$ is a Meyer set if $\Lambda-\Lambda$ is uniformly discrete. Translates $\Lambda$ of arbitrary lattices $L \subset \mathbb{R}^{d}$ are simple examples of Meyer sets, since $\Lambda-\Lambda=L$ is a Delone set. Trivially, any Meyer set is of finite local complexity. Finally, $\Lambda$ is called aperiodic if it has no nonzero translation symmetries.

Definition 2.1 Let $F$ be a finite subset of $\mathbb{R}^{d}$. Furthermore, let $u \in \mathbb{S}^{d-1}$ be a direction, and let $\mathcal{L}_{u}^{d}$ be the set of lines in direction $u$ in $\mathbb{R}^{d}$. Then the (discrete parallel) $X$-ray of $F$ in direction $u$ is the function $X_{u} F: \mathcal{L}_{u}^{d} \rightarrow \mathbb{N}_{0}:=\mathbb{N} \cup\{0\}$ defined by

$$
X_{u} F(\ell):=\operatorname{card}(F \cap \ell)=\sum_{x \in \ell} \mathbb{1}_{F}(x)
$$

Moreover, the support $\left(X_{u} F\right)^{-1}(\mathbb{N})$ of $X_{u} F$, i.e., the set of lines in $\mathcal{L}_{u}^{d}$ which pass through at least one point of $F$, is denoted by $\operatorname{supp}\left(X_{u} F\right)$. Further, for a finite set $U \subset \mathbb{S}^{d-1}$ of directions, set

$$
G_{U}^{F}:=\bigcap_{u \in U}\left(\bigcup_{\ell \in \operatorname{supp}\left(X_{u} F\right)} \ell\right) .
$$

Note that, in the situation of Definition 2.1, one has $F \subset G_{U}^{F}$. Further, $G_{U}^{F}$ is finite if $\operatorname{card} U \geq 2$.

Fact 2.2 Let $h: \mathbb{R}^{d} \rightarrow \mathbb{R}^{d}$ be a homothety, and let $U \subset \mathbb{S}^{d-1}$ be a finite set of directions. Then, one has:

(a) If $P$ is a $U$-polygon, then $h(P)$ is again a $U$-polygon. 
(b) If $F$ and $F^{\prime}$ are finite subsets of $\mathbb{R}^{d}$ with the same $X$-rays in the directions of $U$, then the finite sets $h(F)$ and $h\left(F^{\prime}\right)$ also have the same $X$-rays in the directions of $U$.

Definition 2.3 Let $\mathcal{E}$ be a collection of finite subsets of $\mathbb{R}^{d}$, and let $U \subset \mathbb{S}^{d-1}$ be a finite set of directions. We say that the elements of $\mathcal{E}$ are determined by the $X$-rays in the directions of $U$ if, for all $F, F^{\prime} \in \mathcal{E}$, one has

$$
\left(X_{u} F=X_{u} F^{\prime} \forall u \in U\right) \quad \Longrightarrow \quad F=F^{\prime} .
$$

\section{General Results on Determination}

We need the following property of sets $\Lambda \subset \mathbb{R}^{d}$, where $\langle S\rangle_{\mathbb{Z}}$ denotes the $\mathbb{Z}$-linear hull of a set $S \subset \mathbb{R}^{d}$. In other words, $\langle S\rangle_{\mathbb{Z}}$ is the Abelian group generated by $S$.

(Hom*) For all finite subsets $F$ of $\langle\Lambda-\Lambda\rangle_{\mathbb{Z}}$, there is a homothety

$$
h: \mathbb{R}^{d} \rightarrow \mathbb{R}^{d} \text { such that } h(F) \subset \Lambda .
$$

Translates $\Lambda$ of arbitrary lattices $L \subset \mathbb{R}^{d}$ satisfy $\langle\Lambda-\Lambda\rangle_{\mathbb{Z}}=L$ and are thus Delone sets with property (Hom*). The following negative results shows that, in order to obtain positive results on determination for Delone sets $\Lambda$ with property (Hom*), one has to impose some restriction on the finite subsets of $\Lambda$ to be determined.

Proposition 3.1 Let $\Lambda \subset \mathbb{R}^{d}$ be a Delone set with property $\left(\mathrm{Hom}^{*}\right)$, and let $U$ be a finite set of pairwise nonparallel $\Lambda$-directions. Then the finite subsets of $\Lambda$ are not determined by the $X$-rays in the directions of $U$.

Proof Since property (Hom*) is invariant under translations, we may assume, without loss of generality, that $0 \in \Lambda$. Hence, $\langle\Lambda\rangle_{\mathbb{Z}} \subset\langle\Lambda-\Lambda\rangle_{\mathbb{Z}}$. We argue by induction on card $U$. The case card $U=0$ means $U=\emptyset$ and is obvious. Fix $k \in \mathbb{N}_{0}$ and suppose the assertion is true whenever card $U=k$. Let $U$ now be a set with card $U=k+1$. By the induction hypothesis, there are different finite subsets $F$ and $F^{\prime}$ of $\Lambda$ with the same $X$-rays in the directions of $U^{\prime}$, where $U^{\prime} \subset U$ satisfies card $U^{\prime}=k$. Let $u$ be the remaining direction of $U$ and choose a nonzero element $z \in\langle\Lambda-\Lambda\rangle_{\mathbb{Z}}$ parallel to $u$ such that $z+\left(F \cup F^{\prime}\right)$ and $F \cup F^{\prime}$ are disjoint. Then, $F^{\prime \prime}:=F \cup\left(z+F^{\prime}\right)$ and $F^{\prime \prime \prime}:=F^{\prime} \cup(z+F)$ are different finite subsets of $\langle\Lambda-\Lambda\rangle_{\mathbb{Z}}$ with the same $X$-rays in the directions of $U$. By property (Hom*), there is a homothety $h: \mathbb{R}^{d} \rightarrow \mathbb{R}^{d}$ such that $h\left(F^{\prime \prime} \cup F^{\prime \prime \prime}\right)=h\left(F^{\prime \prime}\right) \cup h\left(F^{\prime \prime \prime}\right) \subset \Lambda$. It follows from Fact 2.2(b) that $h\left(F^{\prime \prime}\right)$ and $h\left(F^{\prime \prime \prime}\right)$ are different finite subsets of $\Lambda$ with the same $X$-rays in the directions of $U$.

Remark 3.2 For other versions of the last result, compare [13, Theorem 4.3.1] and [11, Lemma 2.3.2]. An analysis of the proof of Proposition 3.1 shows that, for any Delone set $\Lambda \subset \mathbb{R}^{d}$ with property (Hom*) and for any finite set $U$ of $k$ pairwise nonparallel $\Lambda$-directions, there are disjoint finite subsets $F$ and $F^{\prime}$ of $\Lambda$ with card $F=\operatorname{card} F^{\prime}=2^{(k-1)}$ that have the same $X$-rays in the directions of $U$. Consider 
Fig. 1 Two contiguous subsets of $\Lambda_{\mathrm{AB}}$ with the same $X$-rays in the two $\Lambda_{\mathrm{AB}}$-directions with slopes 0 and 1 , respectively

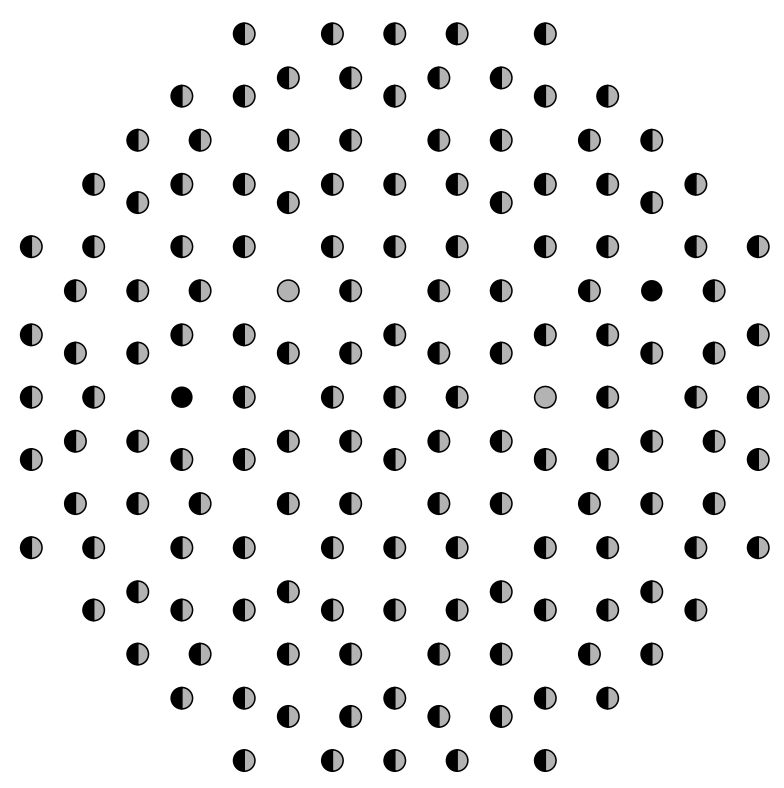

any convex subset $C$ of $\mathbb{R}^{d}$ which contains $F$ and $F^{\prime}$ from above. Then the finite subsets $F_{1}:=(C \cap \Lambda) \backslash F$ and $F_{2}:=(C \cap \Lambda) \backslash F^{\prime}$ of $\Lambda$ also have the same $X$-rays in the directions of $U$. Whereas the points in $F$ and $F^{\prime}$ are widely dispersed over a region, those in $F_{1}$ and $F_{2}$ are contiguous in a way similar to atoms in some solid state material. This procedure is illustrated in Fig. 1 in the case of the aperiodic cyclotomic model set $\Lambda_{\mathrm{AB}}$ as described in Example 4.24 below. Note that cyclotomic model sets have property (Hom*); see Definition 4.1, Remark 4.2 and Proposition 4.31 below.

The subsequent positive results on determination are of limited use in practice since, in general, they do not comply with the restriction to few high-density directions mentioned earlier. The first one is well known and follows from the same arguments as in the proof of [13, Theorem 4.3.3].

Fact 3.3 Let $d \geq 2$, and let $\Lambda$ be a Delone set in $\mathbb{R}^{d}$. Further, let $U$ be any set of $k+1$ pairwise nonparallel $\Lambda$-directions where $k \in \mathbb{N}_{0}$. Then the finite subsets of $\Lambda$ with cardinality less than or equal to $k$ are determined by the $X$-rays in the directions of $U$. Moreover, all finite subsets $F$ of $\Lambda$ with cardinality less than or equal to $k$ satisfy $F=G_{U}^{F}$.

Let $d \geq 2$, and let $\Lambda$ be a Delone set in $\mathbb{R}^{d}$ with property (Hom*). Remark 3.2 and Fact 3.3 show that the finite subsets of $\Lambda$ with cardinality less than or equal to $k$ are determined by the $X$-rays in any set of $k+1$ pairwise nonparallel $\Lambda$-directions but not by the $X$-rays in $1+\left\lfloor\log _{2} k\right\rfloor$ pairwise nonparallel $\Lambda$-directions.

Fact 3.4 Let $d \geq 2$, and let $\Lambda \subset \mathbb{R}^{d}$ be relatively dense. Then the set of $\Lambda$-directions is dense in $\mathbb{S}^{d-1}$. 
Proposition 3.5 Let $d \geq 2$, let $r>0$, and let $\Lambda \subset \mathbb{R}^{d}$ be a Delone set of finite local complexity. Then, there is a set $U$ of two nonparallel $\Lambda$-directions such that the subsets of patches of radius $r$ of $\Lambda$ are determined by the $X$-rays in the directions of $U$. Moreover, there is a set $U$ of three pairwise nonparallel $\Lambda$-directions such that, for all subsets $F$ of patches of radius $r$ of $\Lambda$, one has $F=G_{U}^{F}$.

Proof We denote by $\mathcal{P}_{r}(\Lambda)$ the collection of subsets of patches of radius $r$ of $\Lambda$. For the first assertion, note that the finite local complexity of $\Lambda$ implies that the set $V$ of $\Lambda$-directions $v$ with the property that there is a set $F \in \mathcal{P}_{r}(\Lambda)$ and a line $\ell$ in $\mathbb{R}^{d}$ in direction $v$ with more than one point of $F$ on $\ell$ is finite. Let $u$ be an arbitrary $\Lambda$-direction. Then, for every $F \in \mathcal{P}_{r}(\Lambda)$, one has $F \subset G_{\{u\}}^{F} \cap \Lambda$. Choose $u^{\prime \prime} \in \mathbb{S}^{d-1} \cap$ $u^{\perp}$ and note that, for every $F \in \mathcal{P}_{r}(\Lambda)$, the orthogonal projection $\left(G_{\{u\}}^{F} \cap \Lambda\right) \mid u^{\perp}$ of the set $G_{\{u\}}^{F} \cap \Lambda$ on the hyperplane $u^{\perp}$ is finite with diameter $D_{u}^{F}<2 r$. Moreover, by the finite local complexity of $\Lambda$, the set $\left\{D_{u}^{F} \mid F \in \mathcal{P}_{r}(\Lambda)\right\}$ of diameters is finite. This implies the existence of a neighbourhood $W$ of $u^{\prime \prime}$ in $\mathbb{S}^{d-1}$ with the property that, for each line $\ell$ in a direction $w \in W$ and any set $F \in \mathcal{P}_{r}(\Lambda)$, any two elements of the set $\ell \cap\left(G_{\{u\}}^{F} \cap \Lambda\right)$ have a distance less than $2 r$. Since the set of $\Lambda$-directions is dense in $\mathbb{S}^{d-1}$ by Fact 3.4 , by the finiteness of the set $V$, this observation shows that one can choose a $\Lambda$-direction $u^{\prime} \in W \backslash V$ that is not parallel to $u$. We claim that the elements of $\mathcal{P}_{r}(\Lambda)$ are determined by the $X$-rays in the directions of $\left\{u, u^{\prime}\right\}$. To this end, let $F, F^{\prime} \in \mathcal{P}_{r}(\Lambda)$ satisfy $X_{u} F=X_{u} F^{\prime}$. Then, one has $F, F^{\prime} \subset G_{\{u\}}^{F} \cap \Lambda$. In order to demonstrate that the identity $X_{u^{\prime}} F=X_{u^{\prime}} F^{\prime}$ implies the equality $F=F^{\prime}$, it suffices to show that each line $\ell$ in direction $u^{\prime}$ meets at most one element of $G_{\{u\}}^{F} \cap \Lambda$. Assume the existence of two distinct elements, say $\lambda$ and $\lambda^{\prime}$, in $\ell \cap\left(G_{\{u\}}^{F} \cap \Lambda\right)$. Then, by construction, the distance between $\lambda$ and $\lambda^{\prime}$ is less than $2 r$. Hence, $\left\{\lambda, \lambda^{\prime}\right\} \in \mathcal{P}_{r}(\Lambda)$, and further $u^{\prime} \in V$, a contradiction.

For the second part, let $u, u^{\prime}$ be two arbitrary nonparallel $\Lambda$-directions and set $U^{\prime}:=\left\{u, u^{\prime}\right\}$. Note that the finite local complexity of $\Lambda$ implies that the set $V$ of $\Lambda$-directions $v$ with the property that there is a set $F \in \mathcal{P}_{r}(\Lambda)$ and a line $\ell$ in $\mathbb{R}^{d}$ in direction $v$ with more than one point of the finite set $G_{U^{\prime}}^{F}$ on $\ell$ is finite. Since the set of $\Lambda$-directions is dense in $\mathbb{S}^{d-1}$ by Fact 3.4, this observation shows that one can choose a $\Lambda$-direction $u^{\prime \prime} \notin V$ that is not parallel to $u$ and $u^{\prime}$. By construction, the assertion follows with $U:=\left\{u, u^{\prime}, u^{\prime \prime}\right\}$.

\section{Determination of Convex Subsets of Algebraic Delone Sets}

\subsection{Algebraic Delone Sets}

For $\Lambda \subset \mathbb{C}$, we denote by $\mathbb{K}_{\Lambda}$ the field extension of $\mathbb{Q}$ that is given by

$$
\mathbb{K}_{\Lambda}:=\mathbb{Q}((\Lambda-\Lambda) \cup(\overline{\Lambda-\Lambda})),
$$

and, further, set $\mathbb{k}_{\Lambda}:=\mathbb{K}_{\Lambda} \cap \mathbb{R}$, the maximal real subfield of $\mathbb{K}_{\Lambda}$. The following notion will be useful; see also [22, 24] for generalizations and results related to those presented below. 
Definition 4.1 A Delone set $\Lambda \subset \mathbb{R}^{2}$ is called an algebraic Delone set if it satisfies the following properties:

(Alg) $\quad\left[\mathbb{K}_{\Lambda}: \mathbb{Q}\right]<\infty$.

(Hom) For all finite subsets $F$ of $\mathbb{K}_{\Lambda}$, there is a homothety

$$
h: \mathbb{R}^{2} \rightarrow \mathbb{R}^{2} \text { such that } h(F) \subset \Lambda \text {. }
$$

Translates $\Lambda$ of the square lattice $\mathbb{Z}^{2}=\mathbb{Z}[i]$ are examples of algebraic Delone sets, with $\mathbb{K}_{\Lambda}=\mathbb{Q}(i)$.

Remark 4.2 Note that, for any algebraic Delone set $\Lambda$, the field $\mathbb{k}_{\Lambda}$ is a real algebraic number field. Trivially, property (Hom) for $\Lambda$ implies property (Hom*).

Lagarias [27] defined the notion of finitely generated Delone sets $\Lambda \subset \mathbb{R}^{d}$. These are Delone sets $\Lambda$ with the property that the Abelian group $\langle\Lambda-\Lambda\rangle_{\mathbb{Z}}$ is finitely generated. The last property is always fulfilled by Delone sets of finite local complexity, which are also called Delone sets of finite type; see [27, Theorem 2.1].

Proposition 4.3 Let $\Lambda$ be an algebraic Delone set. If $\Lambda-\Lambda$ is contained in the ring of algebraic integers, then $\Lambda$ is a finitely generated Delone set.

Proof If $\Lambda-\Lambda$ is contained in the ring of algebraic integers, then the Abelian group $\langle\Lambda-\Lambda\rangle_{\mathbb{Z}}$ is a subgroup of the ring of integers in $\mathbb{K}_{\Lambda}$. Since the latter is the maximal order of $\mathbb{K}_{\Lambda}$ and thus a free Abelian group of finite rank, the assertion follows; cf. [8, Chap. 2, Sect. 2].

\subsection{U-polygons in Algebraic Delone Sets}

The following fact follows immediately from property (Hom) in conjunction with Fact 2.2(a).

Fact 4.4 Let $\Lambda$ be an algebraic Delone set and let $U \subset \mathbb{S}^{1}$ be a finite set of directions. Then there is a $U$-polygon in $\mathbb{K}_{\Lambda}$ if and only if there is a $U$-polygon in $\Lambda$.

Lemma 4.5 Let $\Lambda$ be an algebraic Delone set. If $U$ is any set of up to three pairwise nonparallel $\Lambda$-directions, then there exists a $U$-polygon in $\Lambda$.

Proof Without loss of generality, we may assume that card $U=3$. First, construct a triangle in $\mathbb{K}_{\Lambda}$ having sides parallel to the given directions of $U$. If two of the vertices are chosen in $\mathbb{K}_{\Lambda}$, then the third is automatically in $\mathbb{K}_{\Lambda}$. Now, fit six congruent versions of this triangle together in the obvious way to make an affinely regular hexagon in $\mathbb{K}_{\Lambda}$. The latter is then a $U$-polygon in $\mathbb{K}_{\Lambda}$, and the assertion follows from Fact 4.4.

Proposition 4.6 Let $\Lambda$ be an algebraic Delone set, and let $U$ be a set of two or more pairwise nonparallel $\Lambda$-directions. The following statements are equivalent: 
(i) The convex subsets of $\Lambda$ are determined by the $X$-rays in the directions of $U$.

(ii) There is no $U$-polygon in $\Lambda$.

Proof For (i) $\Rightarrow$ (ii), suppose the existence of a $U$-polygon $P$ in $\Lambda$. Partition the vertices of $P$ into two disjoint sets $V, V^{\prime}$, where the elements of these sets alternate round the boundary bd $P$ of $P$. Since $P$ is a $U$-polygon, each line in the plane parallel to some $u \in U$ that contains a point in $V$ also contains a point in $V^{\prime}$. In particular, one sees that card $V=\operatorname{card} V^{\prime}$. Set $C:=(\Lambda \cap P) \backslash\left(V \cup V^{\prime}\right)$. Then, $F:=C \cup V$ and $F^{\prime}:=C \cup V^{\prime}$ are different convex subsets of $\Lambda$ with the same $X$-rays in the directions of $U$.

For (ii) $\Rightarrow$ (i), suppose the existence of two different convex subsets of $\Lambda$ with the same $X$-rays in the directions of $U$. Since the property of being an algebraic Delone set is invariant under translations, we may assume, without loss of generality, that $0 \in \Lambda$, whence $\Lambda \subset \Lambda-\Lambda$. Then, by the same argumentation as in the proof of the corresponding direction of [12, Theorem 5.5], there follows the existence of a $U$-polygon in $\mathbb{Q}(\Lambda) \subset \mathbb{K}_{\Lambda}$. More precisely, one has to use Lemma 4.5 instead of [12, Lemma 4.4] and note that [12, Lemma 5.2] extends to the more general situation needed here. Fact 4.4 completes the proof.

Let $\left(t_{1}, t_{2}, t_{3}, t_{4}\right)$ be an ordered tuple of four pairwise distinct elements of the set $\mathbb{R} \cup\{\infty\}$. Then, its cross ratio $\left\langle t_{1}, t_{2}, t_{3}, t_{4}\right\rangle$ is the nonzero real number defined by

$$
\left\langle t_{1}, t_{2}, t_{3}, t_{4}\right\rangle:=\frac{\left(t_{3}-t_{1}\right)\left(t_{4}-t_{2}\right)}{\left(t_{3}-t_{2}\right)\left(t_{4}-t_{1}\right)},
$$

where one uses the usual conventions if one of the $t_{i}$ equals $\infty$.

Fact 4.7 For a set $\Lambda \subset \mathbb{R}^{2}$, the cross ratio of slopes of four pairwise nonparallel $\Lambda$-directions is an element of the field $\mathbb{k}_{\Lambda}$.

The proof of the following central result uses Darboux's theorem on second midpoint polygons; see $[9,15]$ or [11, Chap. 1].

Theorem 4.8 Let $\Lambda \subset \mathbb{R}^{2}$, let $U$ be a set of four or more pairwise nonparallel $\Lambda$-directions, and suppose the existence of a $U$-polygon. Then the cross ratio of slopes of any four directions of $U$, arranged in order of increasing angle with the positive real axis, is an element of the set

$$
\left(\bigcup_{m \geq 4}\left(\bigcup_{\substack{k_{3}<k_{1} \leq k_{2}<k_{4} \leq m-1 \\ k_{1}+k_{2}=k_{3}+k_{4}}} \frac{\left(1-\zeta_{m}^{k_{1}}\right)\left(1-\zeta_{m}^{k_{2}}\right)}{\left(1-\zeta_{m}^{k_{3}}\right)\left(1-\zeta_{m}^{k_{4}}\right)}\right)\right) \cap \mathbb{k}_{\Lambda},
$$

where $\zeta_{m}:=e^{2 \pi i / m}$, a primitive $m$ th root of unity in $\mathbb{C}$.

Proof The assertion follows from the same arguments as in the first part of the proof of [12, Theorem 4.5]. Here, one additionally has to employ Fact 4.7. 
In order to find a sufficient condition for the determination of the convex subsets of an algebraic Delone set $\Lambda$ by $X$-rays in four pairwise nonparallel $\Lambda$-directions, it is essential for our approach to gain some insight into the set (4.1). This is done in the next section.

\subsection{A Cyclotomic Theorem}

We need the following facts from the theory of algebraic number fields. Let $\mathbb{K} / \mathbb{k}$ be an extension of algebraic number fields (i.e., finite extensions of $\mathbb{Q}$ ) of degree $d:=[\mathbb{K}: \mathbb{k}] \in \mathbb{N}$. Since $\mathbb{K} / \mathbb{k}$ is separable, the corresponding norm $N_{\mathbb{K} / \mathbb{k}}: \mathbb{K} \rightarrow \mathbb{k}$ is given by

$$
N_{\mathbb{K} / \mathbb{k}}(\kappa)=\prod_{j=1}^{d} \sigma_{j}(\kappa),
$$

where the $\sigma_{j}$ are the $d$ distinct embeddings of $\mathbb{K} / \mathbb{k}$ into $\mathbb{C} / \mathbb{k}$; compare [8, Algebraic Supplement, Sect. 2, Corollary 1]. In particular, one has $N_{\mathbb{K} / \mathbb{k}}(\kappa)=\kappa^{d}$ for any $\kappa \in \mathbb{k}$. The norm $N_{\mathbb{K} / \mathbb{k}}$ is multiplicative, i.e., for any $\kappa, \lambda \in \mathbb{K}$, one has

$$
N_{\mathbb{K} / \mathbb{k}}(\kappa \lambda)=N_{\mathbb{K} / \mathbb{k}}(\kappa) N_{\mathbb{K} / \mathbb{k}}(\lambda) .
$$

In particular, the norm $N_{\mathbb{K} / \mathbb{k}}$ induces a homomorphism from the multiplicative subgroup $\mathbb{K}^{\times}$of $\mathbb{K}$ to the multiplicative subgroup $\mathbb{k}^{\times}$of $\mathbb{k}$. Moreover, the norm is transitive in the following sense. If $\mathbb{L}$ is any intermediate field of $\mathbb{K} / \mathbb{k}$ above, then one has

$$
N_{\mathbb{K} / \mathbb{k}}=N_{\mathbb{L} / \mathbb{k}} \circ N_{\mathbb{K} / \mathbb{L}} .
$$

We also need the following facts from the theory of $p$-adic valuations; compare $[16,26]$ for details. Let $p \in \mathbb{P}$. The $p$-adic valuation on $\mathbb{Z}$ is the function $v_{p}$ defined by $v_{p}(0):=\infty$ together with the equation

$$
n=p^{v_{p}(n)} n^{\prime}
$$

for $n \neq 0$, where $p$ does not divide $n^{\prime}$; that is, $v_{p}(n)$ is the exponent of the highest power of $p$ that divides $n$. The function $v_{p}$ is extended to $\mathbb{Q}$ by defining

$$
v_{p}\left(\frac{a}{b}\right):=v_{p}(a)-v_{p}(b)
$$

for $a, b \in \mathbb{Z} \backslash\{0\}$. Note that $v_{p}$ is $\mathbb{Z}$-valued on $\mathbb{Q} \backslash\{0\}$. Further, $v_{p}$ can be extended to the algebraic closure $\mathbb{Q}_{p}^{\text {alg }}$ of the field $\mathbb{Q}_{p}$ of $p$-adic numbers, containing $\mathbb{Q}$. Note that $\mathbb{Q}_{p}^{\text {alg }}$ contains the algebraic closure $\mathbb{Q}^{\text {alg }}$ of $\mathbb{Q}$ and hence all algebraic numbers. On $\mathbb{Q}_{p}^{\text {alg }} \backslash\{0\}, v_{p}$ takes values in $\mathbb{Q}$ and satisfies

$$
\begin{aligned}
& v_{p}(-x)=v_{p}(x), \\
& v_{p}(x y)=v_{p}(x)+v_{p}(y),
\end{aligned}
$$




$$
v_{p}\left(\frac{x}{y}\right)=v_{p}(x)-v_{p}(y)
$$

and

$$
v_{p}(x+y) \geq \min \left\{v_{p}(x), v_{p}(y)\right\} .
$$

For $n \in \mathbb{N}$, we always let $\zeta_{n}:=e^{2 \pi i / n}$, as a specific choice for a primitive $n$th root of unity in $\mathbb{C}$. Further, $\phi$ will always denote Euler's totient function, i.e.,

$$
\phi(n)=\operatorname{card}(\{k \in \mathbb{N} \mid 1 \leq k \leq n \text { and } \operatorname{gcd}(k, n)=1\}) .
$$

Fact 4.9 [12, Proposition 3.6] Let $p \in \mathbb{P}$, and let $r, s, t \in \mathbb{N}$. If $r$ is not a power of $p$ and $\operatorname{gcd}(r, s)=1$, one has

$$
v_{p}\left(1-\zeta_{r}^{s}\right)=0
$$

Otherwise, if $\operatorname{gcd}(p, s)=1$, then

$$
v_{p}\left(1-\zeta_{p^{t}}^{s}\right)=\frac{1}{p^{t-1}(p-1)} .
$$

Definition 4.10 Let $k, m \in \mathbb{N}$, and let $p \in \mathbb{P}$. An $m$ th root of unity $\zeta_{m}^{k}$ is called a $p$-power root of unity if there is a $t \in \mathbb{N}$ such that $\frac{k}{m}=\frac{s}{p^{t}}$ for some $s \in \mathbb{N}$ with $\operatorname{gcd}(p, s)=1$.

Note that an $m$ th root of unity $\zeta_{m}^{k}$ is a $p$-power root of unity if and only if it is a primitive $p^{t}$ th root of unity for some $t \in \mathbb{N}$. The following property is immediate.

Fact 4.11 Let $k, t \in \mathbb{N}$ and $p \in \mathbb{P}$. Further, let $j, m \in \mathbb{N}$ with $\operatorname{gcd}(j, m)=1$. Then, $\zeta_{m}^{k}$ is a primitive $p^{t}$ th root of unity if and only if $\left(\zeta_{m}^{j}\right)^{k}$ is a primitive $p^{t}$ th root of unity.

Fact 4.12 (Gauß) [36, Theorem 2.5] The nth cyclotomic field $\mathbb{Q}\left(\zeta_{n}\right)$ is of degree $\left[\mathbb{Q}\left(\zeta_{n}\right): \mathbb{Q}\right]=\phi(n)$ over $\mathbb{Q}$. The field extension $\mathbb{Q}\left(\zeta_{n}\right) / \mathbb{Q}$ is a Galois extension with Abelian Galois group $G\left(\mathbb{Q}\left(\zeta_{n}\right) / \mathbb{Q}\right) \simeq(\mathbb{Z} / n \mathbb{Z})^{\times}$, where $a(\bmod n)$ with $\operatorname{gcd}(a, n)=1$ corresponds to the automorphism given by $\zeta_{n} \mapsto \zeta_{n}^{a}$.

Lemma 4.13 Let $m, k \in \mathbb{N}$, and let $p \in \mathbb{P}$. If $\sigma \in G\left(\mathbb{Q}\left(\zeta_{m}\right) / \mathbb{Q}\right)$, then

$$
v_{p}\left(1-\zeta_{m}^{k}\right)=v_{p}\left(\sigma\left(1-\zeta_{m}^{k}\right)\right)
$$

Proof By Fact 4.12, $\sigma$ is given by $\zeta_{m} \mapsto \zeta_{m}^{j}$, where $j \in \mathbb{N}$ satisfies $\operatorname{gcd}(j, m)=1$. The assertion follows immediately from Fact 4.9 in conjunction with Fact 4.11.

Definition 4.14 Let $m \geq 4$ be a natural number. We define

$$
D_{m}^{\prime}:=\left\{\left(k_{1}, k_{2}, k_{3}, k_{4}\right) \in \mathbb{N}^{4} \mid k_{1}, k_{2}, k_{3}, k_{4} \leq m-1 \text { and } k_{1}+k_{2}=k_{3}+k_{4}\right\},
$$


together with its subset

$$
D_{m}:=\left\{\left(k_{1}, k_{2}, k_{3}, k_{4}\right) \in \mathbb{N}^{4} \mid k_{3}<k_{1} \leq k_{2}<k_{4} \leq m-1 \text { and } k_{1}+k_{2}=k_{3}+k_{4}\right\},
$$

and define the function $f_{m}: D_{m}^{\prime} \rightarrow \mathbb{Q}\left(\zeta_{m}\right)^{\times}$by

$$
f_{m}\left(\left(k_{1}, k_{2}, k_{3}, k_{4}\right)\right):=\frac{\left(1-\zeta_{m}^{k_{1}}\right)\left(1-\zeta_{m}^{k_{2}}\right)}{\left(1-\zeta_{m}^{k_{3}}\right)\left(1-\zeta_{m}^{k_{4}}\right)}
$$

In fact, the function $f_{m}$ is real valued; see the proof of [12, Lemma 3.1]. Together with Fact 4.12 , this shows that, for any $d \in D_{m}^{\prime}$, the field $\mathbb{Q}\left(f_{m}(d)\right)$ is a real algebraic number field. Without further mention, we shall use this fact in the following.

Lemma 4.15 Let $m \geq 4$, let $p \in \mathbb{P}$, and let $d \in D_{m}^{\prime}$. If $\sigma \in G\left(\mathbb{Q}\left(\zeta_{m}\right) / \mathbb{Q}\right)$, then

$$
v_{p}\left(f_{m}(d)\right)=v_{p}\left(\sigma\left(f_{m}(d)\right)\right) .
$$

Proof The assertion follows from Lemma 4.13 together with (4.5) and (4.6).

Fact 4.16 [28, Chap. 5.2, Theorem 2.8] Let $\sigma: \mathbb{K} \rightarrow \mathbb{K}^{\prime}$ be an isomorphism of fields, let $\mathbb{E}$ be an algebraic extension of $\mathbb{K}$, and let $\mathbb{L}$ be an algebraically closed extension of $\mathbb{K}^{\prime}$. Then, there exists a field homomorphism $\sigma^{\prime}: \mathbb{E} \rightarrow \mathbb{L}$ which extends $\sigma$.

Lemma 4.17 Let $m \geq 4$, and let $d \in D_{m}^{\prime}$. Then, for any prime factor $p \in \mathbb{P}$ of the numerator of the field norm $N_{\mathbb{Q}\left(f_{m}(d)\right) / \mathbb{Q}}\left(f_{m}(d)\right)$, one has

$$
v_{p}\left(N_{\mathbb{Q}}\left(f_{m}(d)\right) / \mathbb{Q}\left(f_{m}(d)\right)\right)=e v_{p}\left(f_{m}(d)\right) \in \mathbb{N},
$$

where $e:=\left[\mathbb{Q}\left(f_{m}(d)\right): \mathbb{Q}\right] \in \mathbb{N}$ is the degree of $f_{m}(d)$ over $\mathbb{Q}$.

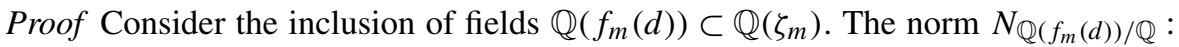
$\mathbb{Q}\left(f_{m}(d)\right) \rightarrow \mathbb{Q}$ of the Galois extension $\mathbb{Q}\left(f_{m}(d)\right) / \mathbb{Q}$ is given by

$$
N_{\mathbb{Q}\left(f_{m}(d)\right) / \mathbb{Q}}(q)=\prod_{j=1}^{e} \sigma_{j}(q)
$$

for $q \in \mathbb{Q}\left(f_{m}(d)\right)$, where $\left\{\sigma_{1}, \ldots, \sigma_{e}\right\}$ is the underlying set of the Galois group $G\left(\mathbb{Q}\left(f_{m}(d)\right) / \mathbb{Q}\right)$. Note that the field extension $\mathbb{Q}\left(f_{m}(d)\right) / \mathbb{Q}$ is indeed a Galois extension since, by Fact 4.12 , the Galois extension $\mathbb{Q}\left(\zeta_{m}\right) / \mathbb{Q}$ has an Abelian Galois group. Moreover, each field automorphism $\sigma_{j} \in G\left(\mathbb{Q}\left(f_{m}(d)\right) / \mathbb{Q}\right)$ can be extended to a field automorphism $\sigma_{j}^{\prime} \in G\left(\mathbb{Q}\left(\zeta_{m}\right) / \mathbb{Q}\right)$ by Fact 4.16. It follows that

$$
N_{\mathbb{Q}\left(f_{m}(d)\right) / \mathbb{Q}}\left(f_{m}(d)\right)=\prod_{j=1}^{e} \sigma_{j}^{\prime}\left(f_{m}(d)\right) .
$$

Using the $p$-adic valuation $v_{p}$ in conjunction with (4.5) and Lemma 4.15, the assertion follows. 
The multiplicativity of norms immediately implies the following:

Fact 4.18 Let $m \geq 4$, and let $d:=\left(k_{1}, k_{2}, k_{3}, k_{4}\right) \in D_{m}^{\prime}$. Then, one has $d^{\prime}:=$ $\left(k_{3}, k_{4}, k_{1}, k_{2}\right) \in D_{m}^{\prime}$ and $f_{m}\left(d^{\prime}\right)=1 / f_{m}(d)$, whence $\mathbb{Q}\left(f_{m}(d)\right)=\mathbb{Q}\left(f_{m}\left(d^{\prime}\right)\right)$. Further, one has the identity

$$
N_{\mathbb{Q}\left(f_{m}(d)\right) / \mathbb{Q}}\left(f_{m}\left(d^{\prime}\right)\right)=\left(N_{\mathbb{Q}\left(f_{m}(d)\right) / \mathbb{Q}}\left(f_{m}(d)\right)\right)^{-1} .
$$

The following main result of this section may be viewed as a generalization of the first part of [12, Theorem 3.10].

Theorem 4.19 For any real algebraic number field $\mathbb{k}$, the set

$$
N_{\mathbb{k} / \mathbb{Q}}\left(\left(\bigcup_{m \geq 4} f_{m}\left(D_{m}\right)\right) \cap \mathbb{k}\right)
$$

is a finite subset of $\mathbb{Q}$.

Proof Set $f:=[\mathbb{k}: \mathbb{Q}] \in \mathbb{N}$ and let $f_{m}(d) \in\left(\bigcup_{m \geq 4} f_{m}\left(D_{m}^{\prime}\right)\right) \cap \mathbb{k}$ for suitable $m \geq 4$ and $d=\left(k_{1}, k_{2}, k_{3}, k_{4}\right) \in D_{m}^{\prime}$. Since $\mathbb{Q}\left(f_{m}(d)\right)$ is an intermediate field of the field extension $\mathbb{k} / \mathbb{Q}, f_{m}(d)$ is of degree $e:=\left[\mathbb{Q}\left(f_{m}(d)\right): \mathbb{Q}\right]$ over $\mathbb{Q}$, where $e$

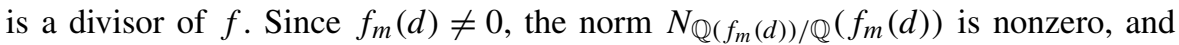
hence its absolute value is greater than zero. Suppose that the absolute value of $N_{\mathbb{Q}\left(f_{m}(d)\right) / \mathbb{Q}}\left(f_{m}(d)\right)$ is greater than 1 . Then the numerator of $N_{\mathbb{Q}\left(f_{m}(d)\right) / \mathbb{Q}}\left(f_{m}(d)\right)$ has a prime factor, say $p \in \mathbb{P}$. Further, by Lemma 4.17, for every such prime factor $p \in \mathbb{P}$, one has

$$
v_{p}\left(N_{\mathbb{Q}\left(f_{m}(d)\right) / \mathbb{Q}}\left(f_{m}(d)\right)\right)=e v_{p}\left(f_{m}(d)\right)=e v_{p}\left(\frac{\left(1-\zeta_{m}^{k_{1}}\right)\left(1-\zeta_{m}^{k_{2}}\right)}{\left(1-\zeta_{m}^{k_{3}}\right)\left(1-\zeta_{m}^{k_{4}}\right)}\right) \in \mathbb{N}
$$

Applying (4.5), (4.6) and Fact 4.9, one sees that $v_{p}\left(f_{m}(d)\right)$ is a sum of at most four terms of the form $1 /\left(p^{t^{\prime}-1}(p-1)\right)$ for various $t^{\prime} \in \mathbb{N}$, with one or two positive terms and at most two negative ones. Let $t$ be the smallest $t^{\prime}$ occurring in one of the positive terms. Then, (4.11) shows in particular that $2 e /\left(p^{t-1}(p-1)\right) \geq 1$ or, equivalently,

$$
p^{t-1}(p-1) \leq 2 e .
$$

One can now see that, by (4.11) and (4.12) and the obvious fact that $p^{t-1}(p-1) \rightarrow$ $\infty$ for fixed $p \in \mathbb{P}$ as $t \rightarrow \infty$ (resp., for fixed $t \in \mathbb{N}$ as $\mathbb{P} \ni p \rightarrow \infty$ ), there are only a finite number of cases to deal with. In particular, one sees that the numerator of $N_{\mathbb{Q}\left(f_{m}(d)\right) / \mathbb{Q}}\left(f_{m}(d)\right)$ can only attain finitely many values. Since, by assumption, the absolute value of $N_{\mathbb{Q}}\left(f_{m}(d)\right) / \mathbb{Q}\left(f_{m}(d)\right)$ is greater than 1 , the existence of a finite set of nonzero rational numbers follows, say $N_{e}$, such that $N_{\mathbb{Q}\left(f_{m}(d)\right) / \mathbb{Q}}\left(f_{m}(d)\right) \in N_{e}$. Moreover, it follows from Fact 4.18 that, if the absolute value of $N_{\mathbb{Q}}\left(f_{m}(d)\right) / \mathbb{Q}\left(f_{m}(d)\right)$ is smaller than one, then one has $N_{\mathbb{Q}\left(f_{m}(d)\right) / \mathbb{Q}}\left(f_{m}(d)\right) \in\left(N_{e}\right)^{-1}$, whereas the missing case can only lead to the norms \pm 1 . The transitivity of norms (cf. (4.3)), in conjunction with the multiplicativity of norms (cf. (4.2)), immediately gives $N_{\mathbb{k} / \mathbb{Q}}\left(f_{m}(d)\right) \in$ 
$\left(\{1,-1\} \cup N_{e} \cup\left(N_{e}\right)^{-1}\right)^{f / e}$. Since $D_{m} \subset D_{m}^{\prime}$ and since the above analysis only depends on the degree $e$ of $f_{m}(d)$ over $\mathbb{Q}$, one obtains

$$
N_{\mathbb{k} / \mathbb{Q}}\left(\left(\bigcup_{m \geq 4} f_{m}\left(D_{m}\right)\right) \cap \mathbb{k}\right) \subset \bigcup_{e \mid f}\left(\{1,-1\} \cup N_{e} \cup\left(N_{e}\right)^{-1}\right)^{\frac{f}{e}}
$$

for suitable finite subsets $N_{e}$ of $\mathbb{Q} \backslash\{0\}$. The assertion follows.

\subsection{Results}

Theorem 4.20 For all algebraic Delone sets $\Lambda$, there is a finite set $N_{\Lambda} \subset \mathbb{Q}$ such that, for all sets $U$ of four or more pairwise nonparallel $\Lambda$-directions, one has the following: If there exists a $U$-polygon, then the cross ratio of slopes of any four directions of $U$, arranged in order of increasing angle with the positive real axis, maps under the norm $N_{\mathbb{k}_{\Lambda} / \mathbb{Q}}$ to $N_{\Lambda}$.

Proof The assertion is an immediate consequence of Remark 4.2 and Theorems 4.8 and 4.19 .

Summing up, we can now state our main result.

Theorem 4.21 Any algebraic Delone set $\Lambda$ has the following properties:

(a) There is a finite set $N_{\Lambda} \subset \mathbb{Q}$ such that, for all sets $U$ of four pairwise nonparallel $\Lambda$-directions, one has the following: If $U$ has the property that the cross ratio of slopes of the directions of $U$, arranged in order of increasing angle with the positive real axis, does not map under the norm $N_{\mathbb{k}_{\Lambda} / \mathbb{Q}}$ to $N_{\Lambda}$, then the convex subsets of $\Lambda$ are determined by the $X$-rays in the directions of $U$.

(b) For all sets $U$ of three or less pairwise nonparallel $\Lambda$-directions, the convex subsets of $\Lambda$ are not determined by the $X$-rays in the directions of $U$.

Proof Part (a) follows immediately from Proposition 4.6 in conjunction with Theorem 4.20. Assertion (b) is an immediate consequence of Proposition 4.6 in conjunction with Lemma 4.5.

\subsection{Application to Cyclotomic Model Sets and an Open Problem}

Following Moody [29], modified along the lines of the algebraic setting of Pleasants [30], we define as follows.

Definition 4.22 Let $n \geq 3$, and let $.^{\star}: \mathbb{Z}\left[\zeta_{n}\right] \rightarrow\left(\mathbb{R}^{2}\right)^{\phi(n) / 2-1}$ be any map of the form $z \mapsto\left(\sigma_{2}(z), \ldots, \sigma_{\phi(n) / 2}(z)\right)$, where the set $\left\{\sigma_{2}, \ldots, \sigma_{\phi(n) / 2}\right\}$ arises from $G\left(\mathbb{Q}\left(\zeta_{n}\right) / \mathbb{Q}\right) \backslash\left\{\mathrm{id},{ }^{-}\right\}$by choosing exactly one automorphism from each pair of complex conjugate ones (cf. Fact 4.12). Then, for any such choice, each translate $\Lambda$ of

$$
\Lambda(W):=\left\{z \in \mathbb{Z}\left[\zeta_{n}\right] \mid z^{\star} \in W\right\}
$$


where $W \subset\left(\mathbb{R}^{2}\right)^{\phi(n) / 2-1}$ is any set with $\emptyset \neq$ int $W \subset W \subset \operatorname{cl}($ int $W)$ and $\operatorname{cl}($ int $W$ ) compact, is a cyclotomic model set with underlying $\mathbb{Z}$-module $\mathbb{Z}\left[\zeta_{n}\right]$. Moreover, .* and $W$ are called the star map and window of $\Lambda$, respectively.

Remark 4.23 In Definition 4.22, we use the convention that, for $\phi(n)=2$ (i.e., $n \in\{3,4,6\}),\left(\mathbb{R}^{2}\right)^{\phi(n) / 2-1}$ is the trivial Abelian group $\{0\}$, and the star map is the zero map. Note that the ring $\mathbb{Z}\left[\zeta_{n}\right]$ is the ring of integers in the $n$th cyclotomic field and is a dense subset of the plane if $n \notin\{3,4,6\}$; cf. [36, Theorem 2.6]. Model sets $\Lambda \subset \mathbb{R}^{d}$ are Meyer sets and have many other fascinating long-range order properties; see $[29,32,33]$ for the general setting and details. Further, a cyclotomic model set $\Lambda$ with underlying $\mathbb{Z}$-module $\mathbb{Z}\left[\zeta_{n}\right]$ is aperiodic if and only if $n \notin\{3,4,6\}$, i.e., the translates of the square (resp., triangular) lattice are the only examples of cyclotomic model sets with nonzero translation symmetries; cf. [7, 20, 21] for more details and properties of (cyclotomic) model sets.

Example 4.24 For an (aperiodic) cyclotomic model set with underlying $\mathbb{Z}$-module $\mathbb{Z}\left[\zeta_{8}\right]$, consider $\Lambda_{\mathrm{AB}}:=\left\{z \in \mathbb{Z}\left[\zeta_{8}\right] \mid z^{\star} \in W\right\}$, where the star map .* is the Galois automorphism in $G\left(\mathbb{Q}\left(\zeta_{8}\right) / \mathbb{Q}\right.$ ), defined by $\zeta_{8} \mapsto \zeta_{8}^{3}$ (cf. Fact 4.12), and the window $W$ is the regular octagon centred at the origin, with vertices in the directions that arise from the 8 th roots of unity by a rotation through $\pi / 8$, and of unit edge length; see $[1,3,10]$. Then $\Lambda_{\mathrm{AB}}$ is associated with the well-known Ammann-Beenker tiling of the plane with squares and rhombi, both having edge length 1; see Fig. 2 for an illustration. Other examples of aperiodic cyclotomic model sets with underlying $\mathbb{Z}$-module $\mathbb{Z}\left[\zeta_{n}\right]$ are the vertex sets of the Tübingen triangle tiling $[5,6](n=5)$ and the shield tiling [10] $(n=12)$; see [20, 21] for details.

The following result on the maximal real subfield $\mathbb{Q}\left(\zeta_{n}+\bar{\zeta}_{n}\right)$ of the $n$th cyclotomic field follows immediately from Fact 4.12.

Fact 4.25 If $n \geq 3$, one has $\left[\mathbb{Q}\left(\zeta_{n}+\bar{\zeta}_{n}\right): \mathbb{Q}\right]=\phi(n) / 2$.

Note that the ring $\mathbb{Z}\left[\zeta_{n}+\bar{\zeta}_{n}\right]$ is the ring of integers in $\mathbb{Q}\left(\zeta_{n}+\bar{\zeta}_{n}\right)$; cf. [36, Proposition 2.16]. A real algebraic integer $\lambda$ is called a Pisot-Vijayaraghavan number ( $P V$-number) if $\lambda>1$ while all (algebraic) conjugates of $\lambda$ have moduli strictly less than 1. The following fact follows from [31, Chap. 1, Theorem 2].

Fact 4.26 For $n \geq 3$, there is a $P V$-number of degree $\phi(n) / 2$ in $\mathbb{Z}\left[\zeta_{n}+\bar{\zeta}_{n}\right]$.

For $n \geq 3$ and $\lambda \in \mathbb{Z}\left[\zeta_{n}+\bar{\zeta}_{n}\right]$, we denote by $m_{\lambda}^{\star}$ the $\mathbb{Z}$-module endomorphism of $\mathbb{Z}\left[\zeta_{n}\right]^{\star}$ which is given by $m_{\lambda}^{\star}\left(z^{\star}\right)=(\lambda z)^{\star}$, where $z \in \mathbb{Z}\left[\zeta_{n}\right]$ and . ${ }^{\star}$ is a star map of a cyclotomic model set with underlying $\mathbb{Z}$-module $\mathbb{Z}\left[\zeta_{n}\right]$.

Lemma 4.27 Let $n \in \mathbb{N} \backslash\{1,2,3,4,6\}$, and let . ${ }^{\star}$ be a star map of a cyclotomic model set with underlying $\mathbb{Z}$-module $\mathbb{Z}\left[\zeta_{n}\right]$. Then, for any $P V$-number $\lambda$ of degree $\phi(n) / 2$ in $\mathbb{Z}\left[\zeta_{n}+\bar{\zeta}_{n}\right]$, a suitable power of $m_{\lambda}^{\star}$ is contractive, i.e., there are $a k \in \mathbb{N}$ and $a$ $\xi \in(0,1)$ such that $\left\|\left(m_{\lambda}^{\star}\right)^{k}\left(z^{\star}\right)\right\| \leq \xi\left\|z^{\star}\right\|$ holds for all $z \in \mathbb{Z}\left[\zeta_{n}\right]$. 

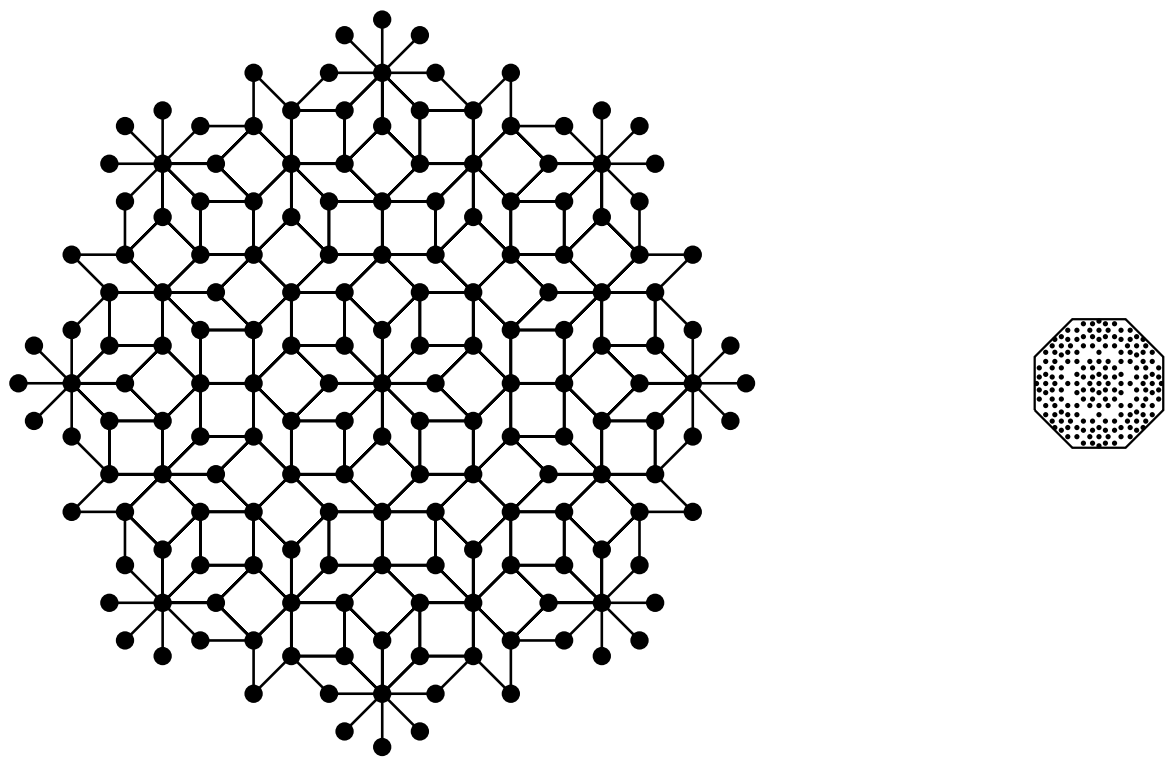

Fig. 2 A central patch of the eightfold Ammann-Beenker tiling with vertex set $\Lambda_{\mathrm{AB}}$ (left) and the .$^{\star}$-image of $\Lambda_{\mathrm{AB}}$ inside the octagonal window (right), with relative scale as described in the text

Proof Since all norms on $\mathbb{R}^{d}$ are equivalent, it suffices to prove the assertion in case of the maximum norm $\|\cdot\|_{\infty}$ on $\left(\mathbb{R}^{2}\right)^{\phi(n) / 2-1}$ with respect to the Euclidean norm on $\mathbb{R}^{2}$ rather than considering the Euclidean norm $\|\cdot\|$ on $\left(\mathbb{R}^{2}\right)^{\phi(n) / 2-1}$ itself. But in that case, the assertion follows immediately with $k:=1$ and

$$
\xi:=\max \left\{\left|\sigma_{j}(\lambda)\right| \mid j \in\{2, \ldots, \phi(n) / 2\}\right\},
$$

since the set $\left\{\lambda, \sigma_{2}(\lambda), \ldots, \sigma_{\phi(n) / 2}(\lambda)\right\}$ equals the set of conjugates of $\lambda$.

Lemma 4.28 Let $n \geq 3$, and let . ${ }^{\star}$ be a star map of a cyclotomic model set with underlying $\mathbb{Z}$-module $\mathbb{Z}\left[\zeta_{n}\right]$. Then $\mathbb{Z}\left[\zeta_{n}\right]^{\star}$ is dense in $\left(\mathbb{R}^{2}\right)^{\phi(n) / 2-1}$.

Proof If $n \in\{3,4,6\}$, one even has $\mathbb{Z}\left[\zeta_{n}\right]^{\star}=\left(\mathbb{R}^{2}\right)^{\phi(n) / 2-1}=\{0\}$. Otherwise, let $\lambda$ be a PV-number of degree $\phi(n) / 2$ in $\mathbb{Z}\left[\zeta_{n}+\bar{\zeta}_{n}\right]$; cf. Fact 4.26 . Then, since $\mathbb{Z}\left[\zeta_{n}\right]$ is the maximal order of $\mathbb{Q}\left(\zeta_{n}\right)$ by Remark 4.23 , for any $k \in \mathbb{N}$, the set

$$
\left\{\left(\lambda^{k} z,\left(m_{\lambda}^{\star}\right)^{k}\left(z^{\star}\right)\right) \mid z \in \mathbb{Z}\left[\zeta_{n}\right]\right\}
$$

is a (full) lattice in $\mathbb{R}^{2} \times\left(\mathbb{R}^{2}\right)^{\phi(n) / 2-1}$; cf. [8, Chap. 2, Sect. 3]. In conjunction with Lemma 4.27 , this implies that, for any $\varepsilon>0$, the $\mathbb{Z}$-module $\mathbb{Z}\left[\zeta_{n}\right]^{\star}$ contains an $\mathbb{R}$-basis of $\left(\mathbb{R}^{2}\right)^{\phi(n) / 2-1}$ whose elements have norms $\leq \varepsilon$. The assertion follows.

Lemma 4.29 Let $n \geq 3$, and let $\Lambda$ be a cyclotomic model set with underlying $\mathbb{Z}$-module $\mathbb{Z}\left[\zeta_{n}\right]$. Then, for any finite set $F \subset \mathbb{Q}\left(\zeta_{n}\right)$, there is a homothety $h: \mathbb{R}^{2} \rightarrow$ $\mathbb{R}^{2}$ such that $h(F) \subset \Lambda$. 
Proof Without loss of generality, we may assume that $\Lambda$ is of the form $\Lambda(W)$ (see Definition 4.22) and, further, that $F \neq \emptyset$. By [28, Chap. 7.1, Proposition 1.1] in conjunction with Fact 4.12 and Remark 4.23, there is an $l \in \mathbb{N}$ with $l F \subset \mathbb{Z}\left[\zeta_{n}\right]$. Let .* be the star map that is used in the construction of $\Lambda(W)$. If $n \in\{3,4,6\}$, we are done by defining the homothety $h: \mathbb{R}^{2} \rightarrow \mathbb{R}^{2}$ by $z \mapsto l z$. Otherwise, by int $W \neq \emptyset$ in conjunction with Lemma 4.28, there follows the existence of a suitable $z_{0} \in \mathbb{Z}\left[\zeta_{n}\right]$ with $z_{0}^{\star} \in$ int $W$. Consider the open neighbourhood $V:=$ int $W-z_{0}^{\star}$ of 0 in $\left(\mathbb{R}^{2}\right)^{\phi(n) / 2-1}$. Next, choose a PV-number $\lambda$ of degree $\phi(n) / 2$ in $\mathbb{Z}\left[\zeta_{n}+\bar{\zeta}_{n}\right]$; cf. Fact 4.26 . By virtue of Lemma 4.27 , there is a $k \in \mathbb{N}$ such that

$$
\left(m_{\lambda}^{\star}\right)^{k}\left((l F)^{\star}\right) \subset V .
$$

It follows that $\left\{\left(\lambda^{k} z+z_{0}\right)^{\star} \mid z \in l F\right\} \subset$ int $W$ and, further, that $h(F) \subset \Lambda(W)$, where $h: \mathbb{R}^{2} \rightarrow \mathbb{R}^{2}$ is the homothety given by $z \mapsto\left(l \lambda^{k}\right) z+z_{0}$.

Fact 4.30 Let $n \geq 3$, and let $\Lambda$ be a cyclotomic model set with underlying $\mathbb{Z}$-module $\mathbb{Z}\left[\zeta_{n}\right]$. Then, one has $\mathbb{K}_{\Lambda} \subset \mathbb{Q}\left(\zeta_{n}\right)$ and thus $\mathbb{k}_{\Lambda} \subset \mathbb{Q}\left(\zeta_{n}+\bar{\zeta}_{n}\right)$.

Proposition 4.31 Cyclotomic model sets are algebraic Delone sets.

Proof Any cyclotomic model set is a Meyer set by Remark 4.23. Properties (Alg) and (Hom) follow immediately from Fact 4.30 in conjunction with Fact 4.12 and Lemma 4.29, respectively.

As another immediate consequence of Lemma 4.29, one verifies the following:

Fact 4.32 Let $n \geq 3$, and let $\Lambda$ be a cyclotomic model set with underlying $\mathbb{Z}$-module $\mathbb{Z}\left[\zeta_{n}\right]$. Then the set of $\Lambda$-directions is precisely the set of $\mathbb{Z}\left[\zeta_{n}\right]$-directions.

An analysis of the proof of Theorem 4.21 gives the following:

Theorem 4.33 For all $n \geq 3$, there is a finite set $N_{n} \subset \mathbb{Q}$ such that, for all cyclotomic model sets $\Lambda$ with underlying $\mathbb{Z}$-module $\mathbb{Z}\left[\zeta_{n}\right]$ and all sets $U$ of four pairwise nonparallel $\mathbb{Z}\left[\zeta_{n}\right]$-directions, one has the following: If $U$ has the property that the cross ratio of slopes of the directions of $U$, arranged in order of increasing angle with the positive real axis, does not map under the norm $N_{\mathbb{Q}\left(\zeta_{n}+\bar{\zeta}_{n}\right) / \mathbb{Q}}$ to $N_{n}$, then the convex subsets of $\Lambda$ are determined by the $X$-rays in the directions of $U$.

The above analysis allows the construction of specific sets $U$ of four pairwise nonparallel $\mathbb{Z}\left[\zeta_{n}\right]$-directions having the property that, for all cyclotomic model sets $\Lambda$ with underlying $\mathbb{Z}$-module $\mathbb{Z}\left[\zeta_{n}\right]$, the convex subsets of $\Lambda$ are determined by the corresponding $X$-rays. For example, the sets of $\mathbb{Z}\left[\zeta_{n}\right]$-directions parallel to the elements of the following sets have this property: $U_{n}:=\left\{1,1+\zeta_{n}, 1+2 \zeta_{n}, 1+5 \zeta_{n}\right\}$, $U_{n}^{\prime}:=\left\{1,2+\zeta_{n}, \zeta_{n},-1+2 \zeta_{n}\right\}$ and $U_{n}^{\prime \prime}:=\left\{2+\zeta_{n}, 3+2 \zeta_{n}, 1+\zeta_{n}, 2+3 \zeta_{n}\right\}$; cf. [20, Theorem 2.54] or [21, Theorem 15] and compare [12, Theorem 5.7 and 
Remark 5.8]. Often, one can even find examples that yield dense lines in the corresponding discrete structures. For example, for the practically relevant quasicrystallographic case of (aperiodic) cyclotomic model sets $\Lambda$ with underlying $\mathbb{Z}$-module $\mathbb{Z}\left[\zeta_{n}\right]$, where $n=5,8,10,12$, this is achieved by the sets of $\mathbb{Z}\left[\zeta_{n}\right]$-directions parallel to the elements of the following sets, where $\tau$ denotes the golden ratio (i.e., $\tau=$ $(1+\sqrt{5}) / 2): U_{8}:=\left\{1+\zeta_{8},(-1+\sqrt{2})+\sqrt{2} \zeta_{8},(-1-\sqrt{2})+\zeta_{8},-2+(-1+\sqrt{2}) \zeta_{8}\right\}$, $U_{5}:=U_{10}:=\left\{(1+\tau)+\zeta_{5},(\tau-1)+\zeta_{5},-\tau+\zeta_{5}, 2 \tau-\zeta_{5}\right\}$ and $U_{12}:=\{1,2+$ $\left.\zeta_{12}, \zeta_{12}, \sqrt{3}-\zeta_{12}\right\}$, respectively; cf. [20, Theorem 2.56, Example 2.57 and Remark 2.58] or [21, Theorem 16, Example 3 and Remark 40]. Note that orders 5, 8, 10 and 12 occur as standard cyclic symmetries of genuine quasicrystals; cf. [35].

Problem 4.34 In the crystallographic case of the square lattice $\mathbb{Z}[i]$, Gardner and Gritzmann were able to show that the convex subsets of $\mathbb{Z}[i]$ are determined by the $X$-rays in the directions of any set $U$ of seven pairwise nonparallel $\mathbb{Z}[i]$ directions; cf. [12, Theorem 5.7]. It would be interesting to know if, for all cyclotomic model sets $\Lambda$ with underlying $\mathbb{Z}$-module $\mathbb{Z}\left[\zeta_{n}\right]$, there exists a natural number $k \in \mathbb{N}$ such that the convex subsets of $\Lambda$ are determined by the $X$-rays in the directions of any set $U$ of $k$ pairwise nonparallel $\mathbb{Z}\left[\zeta_{n}\right]$-directions. Assuming an affirmative answer, a weak relation between $n$ and $k$ was demonstrated in [22, Corollary 5.5].

\section{Final Remark}

For a summary of results for model sets associated with the famous Penrose tiling of the plane, see [2]. These so-called Penrose model sets can also be seen to be algebraic Delone sets. The algorithmic reconstruction problem of discrete tomography of cyclotomic model sets has been studied in [7]. In [23], it is shown how the results for the planar case obtained in [7] and the present text can be lifted to the practically relevant case of so-called icosahedral model sets in $\mathbb{R}^{3}$. For a completer overview of both uniqueness and computational complexity results in the discrete tomography of Delone sets with long-range order, we refer the reader to [20]. This reference also contains results on the interactive concept of successive determination of finite sets by $X$-rays and further extensions of settings and results that are beyond our scope here; compare also [18, 21] and [23].

Acknowledgements The author is indebted to Michael Baake, Richard J. Gardner and Peter A.B. Pleasants for their cooperation and for useful hints on the manuscript. Valuable discussions with Uwe Grimm, Peter Gritzmann and Barbara Langfeld are gratefully acknowledged.

\section{References}

1. Ammann, R., Grünbaum, B., Shephard, G.C.: Aperiodic tiles. Discrete Comput. Geom. 8, 1-25 (1992)

2. Baake, M., Huck, C.: Discrete tomography of Penrose model sets. Philos. Mag. 87, 2839-2846 (2007). arXiv:math-ph/0610056v1 
3. Baake, M., Joseph, D.: Ideal and defective vertex configurations in the planar octagonal quasilattice. Phys. Rev. B 42, 8091-8102 (1990)

4. Baake, M., Moody, R.V. (eds.): Directions in Mathematical Quasicrystals. CRM Monograph Series, vol. 13. AMS, Providence (2000)

5. Baake, M., Kramer, P., Schlottmann, M., Zeidler, D.: The triangle pattern-a new quasiperiodic tiling with fivefold symmetry. Mod. Phys. Lett. B 4, 249-258 (1990)

6. Baake, M., Kramer, P., Schlottmann, M., Zeidler, D.: Planar patterns with fivefold symmetry as sections of periodic structures in 4-space. Int. J. Mod. Phys. B 4, 2217-2268 (1990)

7. Baake, M., Gritzmann, P., Huck, C., Langfeld, B., Lord, K.: Discrete tomography of planar model sets. Acta Crystallogr. A 62, 419-433 (2006). arXiv:math/0609393v1 [math.MG]

8. Borevich, Z.I., Shafarevich, I.R.: Number Theory. Academic Press, San Diego (1966)

9. Darboux, M.G.: Sur un problème de géométrie élémentaire. Bull. Sci. Math. 2, 298-304 (1878)

10. Gähler, F.: Matching rules for quasicrystals: the composition-decomposition method. J. Non-Cryst. Solids 153-154, 160-164 (1993)

11. Gardner, R.J.: Geometric Tomography, 2nd edn. Cambridge University Press, Cambridge (2006)

12. Gardner, R.J., Gritzmann, P.: Discrete tomography: determination of finite sets by X-rays. Trans. Am. Math. Soc. 349, 2271-2295 (1997)

13. Gardner, R.J., Gritzmann, P.: Uniqueness and complexity in discrete tomography. In: [19], pp. 85-114

14. Gardner, R.J., Gritzmann, P., Prangenberg, D.: On the computational complexity of reconstructing lattice sets from their X-rays. Discrete Math. 202, 45-71 (1999)

15. Gardner, R.J., McMullen, P.: On Hammer's X-ray problem. J. Lond. Math. Soc. (2) 21, 171-175 (1980)

16. Gouvêa, F.Q.: p-adic Numbers. Springer, New York (1993)

17. Gritzmann, P.: On the reconstruction of finite lattice sets from their X-rays. In: Ahronovitz, E., Fiorio, C. (eds.) Lecture Notes on Computer Science, pp. 19-32. Springer, London (1997)

18. Gritzmann, P., Langfeld, B.: On the index of Siegel grids and its application to the tomography of quasicrystals. Eur. J. Comb. 29, 1894-1909 (2008)

19. Herman, G.T., Kuba, A. (eds.): Discrete Tomography: Foundations, Algorithms, and Applications. Birkhäuser, Boston (1999)

20. Huck, C.: Discrete tomography of delone sets with long-range order. Ph.D. thesis (Universität Bielefeld). Logos Verlag, Berlin (2007)

21. Huck, C.: Uniqueness in discrete tomography of planar model sets, notes (2007). arXiv:math/ 0701141v2 [math.MG]

22. Huck, C.: A note on affinely regular polygons. Eur. J. Comb. 30(2), 387-395 (2009). arXiv:0801. 3218v1 [math.MG]

23. Huck, C.: Discrete tomography of icosahedral model sets. Acta Crystallogr. A 65, 240-248 (2009). arXiv:0705.3005v2 [math.MG]

24. Huck, C.: On the existence of $U$-polygons of class $c \geq 4$ in planar point sets. Discrete Math. (in press). arXiv:0811.3546v1 [math.MG]

25. Kisielowski, C., Schwander, P., Baumann, F.H., Seibt, M., Kim, Y., Ourmazd, A.: An approach to quantitative high-resolution transmission electron microscopy of crystalline materials. Ultramicroscopy 58, 131-155 (1995)

26. Koblitz, N.: $p$-adic Numbers, $p$-adic Analysis, and Zeta-Functions, 2nd edn. Springer, New York (1984)

27. Lagarias, J.C.: Geometric models for quasicrystals I. Delone sets of finite type. Discrete Comput. Geom. 21(2), 161-191 (1999)

28. Lang, S.: Algebra, 3rd edn. Addison-Wesley, Reading (1993)

29. Moody, R.V.: Model sets: a survey. In: Axel, F., Dénoyer, F., Gazeau, J.-P. (eds.) From Quasicrystals to More Complex Systems, pp. 145-166. EDP Sciences, Les Ulis, and Springer, Berlin (2000). arXiv:math/0002020v1 [math.MG]

30. Pleasants, P.A.B.: Designer quasicrystals: cut-and-project sets with pre-assigned properties. In: [4], pp. 95-141

31. Salem, R.: Algebraic Numbers and Fourier Analysis. Heath, Boston (1963)

32. Schlottmann, M.: Cut-and-project sets in locally compact Abelian groups. In: Patera, J. (ed.) Quasicrystals and Discrete Geometry. Fields Institute Monographs, vol. 10, pp. 247-264. AMS, Providence (1998)

33. Schlottmann, M.: Generalized model sets and dynamical systems. In: [4], pp. 143-159 
34. Schwander, P., Kisielowski, C., Seibt, M., Baumann, F.H., Kim, Y., Ourmazd, A.: Mapping projected potential, interfacial roughness, and composition in general crystalline solids by quantitative transmission electron microscopy. Phys. Rev. Lett. 71, 4150-4153 (1993)

35. Steurer, W.: Twenty years of structure research on quasicrystals. Part I. Pentagonal, octagonal, decagonal and dodecagonal quasicrystals. Z. Kristallogr. 219, 391-446 (2004)

36. Washington, L.C.: Introduction to Cyclotomic Fields, 2nd edn. Springer, New York (1997) 